\title{
Effects of Acupuncture for Anorexia and Dietary Intake Loss in Cachexia Patients With Advanced Respiratory Illness (Pilot Study)
}

\section{Tomonori Maekura}

National Hospital Organisation Toneyama National Hospital: Kokuritsu Byoin Kiko Osaka Toneyama Iryo Center

Ryoji Maekura ( $\nabla_{\text {r-maekura@ghsj.ac.jp) }}$

Graduate School of Health Care Sciences,Jikei Institute https://orcid.org/0000-0003-0788-1157

\section{Keisuke Miki}

National Hospital Organisation Toneyama National Hospital: Kokuritsu Byoin Kiko Osaka Toneyama Iryo Center

\section{Mari Miki}

National Hospital Organisation Toneyama National Hospital: Kokuritsu Byoin Kiko Osaka Toneyama Iryo Center

\section{Seigo Kitada}

National Hospital Organisation Toneyama National Hospital: Kokuritsu Byoin Kiko Osaka Toneyama Iryo Center

\section{Research}

Keywords: Acupuncture, cachexia, chronic lung disease, Lymphgioleiomyomatosis, norepinephrine, ghrelin

Posted Date: August 3rd, 2021

DOI: https://doi.org/10.21203/rs.3.rs-752416/v1

License: (c) (1) This work is licensed under a Creative Commons Attribution 4.0 International License. Read Full License 


\section{Abstract}

Background: Weight loss and low body mass index with cachexia are serious and refractory clinical conditions in patients with advanced chronic respiratory disease. This pilot study aimed to determine whether acupuncture improves dietary intake through increased appetite stimulation, and nutritional biomarkers, in cachectic patients with advanced respiratory illness.

Methods: This study used a single-arm, open experimental design, and was conducted among hospitalized patients whose dietary intake was extremely decreased due to poor appetite. Food intake, and biomarkers were evaluated before and after acupuncture (3 times a week for 3 weeks).

Results: Ten patients (age: 70.5 years [interquartile range (IQR): 68.7-75.8 years], 50\% females) completed the acupuncture treatment. Significant improvements in appetite loss (visual analog scale [VAS]: $58.6 \mathrm{~mm}$ [42-83 mm] to $37.2 \mathrm{~mm}$ [19.5-50.8 mm], p = 0.0039), amount of food intake (1461 $\mathrm{Kcal} / \mathrm{day}$ [1204-1620 Kcal/day] to $1696 \mathrm{Kcal} / \mathrm{day}$ [1443-2047 Kcal/day], $\mathrm{p}=0.0039$ ), and serum nutritional biomarker (albumin: $3.3 \mathrm{~g} / \mathrm{dL}$ [3-3.8 g/dL] to 3.5 [3.3-3.7 g/dL], p = 0.0293; pre-albumin: $14.4 \mathrm{mg} / \mathrm{dL}$ [10-19.5 mg/dL] to $16.7 \mathrm{mg} / \mathrm{dL}$ [11.1-21.6 mg/dL], $\mathrm{p}=0.0137$ ) were observed after acupuncture. Body weight loss ( $0 \mathrm{~kg}[-1.4$ to $1.75 \mathrm{~kg}], \mathrm{p}=0.0078,0 \%$ [-3.25\% to $4.7 \%], \mathrm{p}=0.0078) 6$ months after acupuncture was significantly improved versus 6 months before acupuncture (-3.8 $\mathrm{kg}[-6.4$ to $-0.3 \mathrm{~kg}],-5.2 \%[-11.8 \%$ to $-0.83 \%])$.

Conclusions: Short-term acupuncture may be a novel and safe treatment for improving the appetite and nutritional status of cachectic patients with advanced chronic respiratory illness.

Trial registration: UMIN000044859; Date of registration: July 14, 2021-Retrospectively registered; https://www.umin.ac.jp/

\section{Introduction}

Body weight loss and low body mass index (BMI) with cachexia are serious clinical conditions experienced by patients with chronic advanced diseases [1]. These signs are also observed at the terminal stage of many patients with chronic respiratory diseases (e.g., chronic obstructive pulmonary disease [COPD], Mycobacterium avium complex pulmonary disease [MAC-PD], and idiopathic pulmonary fibrosis [IPF]) [2-5]. Cachexia can also develop in association with many infections. A low BMI is an independent predictor of death due to dyspnea, partial arterial pressure of oxygen, forced expiratory volume in 1 second at rest, and three pathophysiological conditions (hypoxemia, sympathetic overactivity, and acidosis) during exercise among patients with COPD [2, 6].

A nutritional intervention combined with an exercise program may be the most effective treatment for undernourished patients [7]. In Japan, the majority of hospitalized patients generally undergo nutritional therapy by the Nutrition Support Team, which is supported by Japanese healthcare insurance. Ghrelin is the only known circulating orexigenic hormone. In a previous study with cachectic COPD patients, we explored the synergistic effectiveness of 3-week ghrelin administration and pulmonary rehabilitation, including nutritional therapy and exercise training [8]. However, ghrelin and placebo treatment did not achieve a significant improvement in walking distance and weight at the completion of the intervention. Some patients with weight loss were observed after the first week of intervention, and this result may have reduced the effectiveness of ghrelin administration and pulmonary rehabilitation. Excessive exercise training may worsen anabolic and catabolic balance in patients with severely limited exercise tolerance, due to exposure to hypoxemia, sympathetic overactivity (high level of plasma norepinephrine), and acidosis [6, 9].

Acupuncture treatment has been shown to improve feeding behavior, as well as dyspnea during exercise, by improving the fatigue of respiratory accessory muscles and reducing obstructive ventilatory impairment [10]. Breathlessness is also improved due to the reduction of energy consumption during exercise, and this improvement of dyspnea during eating may result in an increased dietary intake [11]. Previous research also indicates that acupuncture is a safe technique and provides benefits to patients with cancer or diabetic gastroparesis by reducing gastrointestinal symptoms and altering the physiological functions of hormones (ghrelin) that play important roles in gastrointestinal functions [12-16].

We conducted a preliminary study to evaluate two hypotheses: a 3-week course of acupuncture therapy improves (1) dietary intake through increased appetite stimulation, and (2) nutritional biomarkers in patients with advanced respiratory illness and severe anorexia.

\section{Methods}

\section{Study design}

This study utilized a single-arm, open experimental design, because it may include patients who are in too severe conditions to randomize cases and have no other applicable treatment. The effects of acupuncture (three times a week for 3 weeks) on appetite, dietary intake, and nutritional biomarkers were evaluated among cachectic patients hospitalized with advanced respiratory diseases, whose dietary intake was significantly decreased due to poor appetite.

\section{Patient recruitment}

Participants were recruited from among those admitted to the respiratory specialized ward of the National Hospital Organization Osaka Toneyama Medical Center between October 1, 2016, and June 30, 2017. Inclusion criteria included patients (>21 years) with (1) severe chronic respiratory diseases (e.g., COPD, MAC-PD, IPF, and lymphangioleiomyomatosis [LAM]); (2) extremely reduced dietary intake due to poor appetite, despite receiving nutritional therapy; and (3) body weight loss of more than $5 \%$ in the last 6 months or $\mathrm{BMI}<21 \mathrm{~kg} / \mathrm{m}^{2}$; and (4) ability to sign informed consent without assistance. Patients with 1 ) malignant tumors; 2) severe heart diseases; 3) hepatic dysfunction; 4) renal failure; 5) a change in drug regimen during acupuncture intervention; or 6 ) an inability to participate, as determined by their physician, were excluded. 


\section{Acupuncture treatment}

The standardized selection of acupuncture points was based on the results of previous studies [10,11], in which the effects on chronic respiratory diseases for the past 5 years were confirmed through clinical experience. The standardized acupuncture points were as follows: 1) Zhongwan of the conception vessel (CV 12); 2) Zusanli 36 of the meridian of the stomach (ST 36); and 3) Pishu of the meridian of the bladder (BL 20). Additional appropriate points were added for other symptoms: 4) LU1 (Zhongfu) and 5) LU-9 (Taiyuan) in the lung meridian for breathlessness; and 6) KD-7 (Fuliu) in the kidney meridian for peripheral edema. Acupuncture treatment is described in detail elsewhere $[10,11,16]$. Therapeutic acupuncture needles (stainless steel; disposable; length, $40 \mathrm{~mm}$; thickness, $0.16 \mathrm{~mm}$; SEIRIN, Shizuoka, Japan). The needle was inserted to a depth of 5-15 mm and rotated gradually for 10 seconds to $1 \mathrm{minute}$ manually throughout the treatment period. Perception of de qi (i.e. tingling, numbness, heaviness, and other feelings) was confirmed after needle insertion and manipulation. The acupuncture was carried out by an acupuncturist with more than five years of clinical experience in respiratory diseases. All patients were followed up during routine medical care after the 3-week acupuncture treatment.

\section{Appetite assessment}

Appetite was assessed as the mean score measured at nine pre-meals for 3 consecutive days, before and after the acupuncture intervention, using a visual analog scale (VAS), which is a 100-mm line ranging from one extreme on the left side ("very good appetite," 0 points) to another extreme on the right side ("no appetite," 100 points) [17]. Patients were asked to report their degree of appetite, which integrated the senses of hunger, fullness, nausea, and breathlessness, by making a vertical mark at the corresponding point on the VAS line. Each score was determined by measuring the distance from the left side of the line to the vertical mark.

\section{Dietary intake}

Food intake was assessed as the mean amount of food consumed for 3 consecutive days, before and after the acupuncture intervention, as described previously [18]. The food intake (Kcal/day), which was averaged over 3 days, was measured by a nutritionist who calculated the daily caloric intake from the patients' electronic medical records (Fujitsu, HOPE/EGMAI-GX). Body weight was measured weekly.

\section{Biomarkers}

Blood samples were collected in the ward for the assessment of systemic inflammation (C-reactive protein [CRP]), nutrition status (albumin [Alb], prealbumin [Pre-Alb], retinol-binding protein), lactate dehydrogenase, appetite hormone (ghrelin), and catecholamines (norepinephrine, epinephrine, and dopamine).

Twenty-four-hour urinary catecholamine levels were measured. Blood specimens were taken from the antecubital vein after a 30-min bed rest in the morning under fasting conditions, and immediately stored on ice and transported to the laboratory for analysis. Samples for ghrelin assessment remained stored at $-80^{\circ} \mathrm{C}$ until all samples were analyzed together in one batch [19]. Biological markers were determined before and after the 3-week acupuncture intervention.

\section{Outcomes}

The primary outcomes were the amount of food intake and VAS score for appetite. Secondary outcomes were nutrition status (Alb, Pre-Alb, retinol-binding protein), appetite hormone (ghrelin), catecholamines (norepinephrine, epinephrine, and dopamine), and 24-hour urinary catecholamine levels.

\section{Statistical analysis}

All data are presented as median and interquartile range (IQR). For the comparisons of parameters before and after acupuncture, we used a Wilcoxon signed-rank test. The relationships between parameters were assessed using Spearman's rank correlation coefficients. A p-value $<0.05$ was considered statistically significant (JMP software, version 11; SAS Institute Inc., NC, United States).

\section{Results}

\section{Study population}

Ten patients met the defined criteria, agreed to participate, and underwent acupuncture. The main respiratory diagnoses were refractory MAC-PD in five cases, COPD in two cases, LAM in two cases, and IPF in one case. The diagnosis, treatment, and prognosis of the participating inpatients are shown in Table 1. Gastrointestinal symptoms, body weight status, degree of breathlessness, and pulmonary function data in patients with severe chronic respiratory illness are shown in Table 2. All patients complained of anorexia (appetite loss: 49 [IQR: 42 to 83]) due to severe gastrointestinal symptoms and breathlessness during eating. Their BMI was very low (15.2 [IQR: 13.9 to 18.7]), and body weight loss over the last 6 months was 5.2\% [IQR: 0.8 to 11.8] (fIve patients had a loss of over $5 \%$ ). The results of the spirometry test, performed before the intervention, revealed severe mixed disorders in five patients and severe restrictive disorders in four patients. The subjects consisted of patients with very severe respiratory diseases. Six patients died before the 3-year follow-up after acupuncture for 3 weeks (survival time: 1.8 [IQR: 0.9 to 2.8] years). All treatments, including nutritional therapy, remained unchanged throughout the study period. 
Table 1

Diagnosis, treatments, and prognosis in participating inpatients

\begin{tabular}{|c|c|c|c|c|c|c|c|c|c|c|}
\hline & & & Diagnosis & & & Treatments & & & & \\
\hline $\begin{array}{l}\text { Case } \\
\text { No. }\end{array}$ & Age & Sex & $\begin{array}{l}\text { Respiratory } \\
\text { diagnosis }\end{array}$ & Comorbidity & $\begin{array}{l}\text { Resection } \\
\text { history }\end{array}$ & Medication & Chemotherapy & $\begin{array}{l}\text { Other } \\
\text { treatments }\end{array}$ & $\begin{array}{l}\text { Survival } \\
\text { time } \\
\text { (years) }\end{array}$ & Prognosis \\
\hline 1 & 69 & Female & $\begin{array}{l}\text { MAC-PD, } \\
\text { COP, } \\
\text { mycosis }\end{array}$ & & $\begin{array}{l}\text { Lobectomy for } \\
\text { LC }\end{array}$ & Prednisolone & $\begin{array}{l}\text { RFP, STFX, } \\
\text { TOB }\end{array}$ & & 1.9 & Nonsurvival $^{\mathrm{a}}$ \\
\hline 2 & 69 & Female & $\begin{array}{l}\text { LAM, PH, } \\
\text { asthma }\end{array}$ & & & $\begin{array}{l}\text { LAMA, LABA, } \\
\text { ICS }\end{array}$ & & $\begin{array}{l}\text { LTOT + } \\
\text { NIV }\end{array}$ & 3.2 & Survival \\
\hline 3 & 56 & Male & COPD & HT & & LAMA, PPI & CAM & LTOT & 3.2 & Survival \\
\hline 4 & 84 & Female & MAC-PD, BE & & $\begin{array}{l}\text { Gastrectomy } \\
\text { for GC }\end{array}$ & $\begin{array}{l}\text { Thyroid } \\
\text { hormone }\end{array}$ & CAM & & 0.4 & Transfer \\
\hline 5 & 72 & Male & MAC-PD & DM & $\begin{array}{l}\text { Gastrectomy } \\
\text { for GU }\end{array}$ & & $\begin{array}{l}\text { CAM, RFP, EB, } \\
\text { LVFX }\end{array}$ & & 1 & Nonsurvival $^{\mathrm{a}}$ \\
\hline 6 & 68 & Female & LAM, PH & DM & & Acarbose & & LTOT & 1.7 & Nonsurvival $^{\circ}$ \\
\hline 7 & 73 & Female & $\begin{array}{l}\text { MAC-PD, } \\
\text { Pyothorax }\end{array}$ & HT & $\begin{array}{l}\text { Lobectomy for } \\
\text { MAC-PD }\end{array}$ & $\mathrm{H} 2$ blocker & $\begin{array}{l}\text { AZM, RBT } \\
\text { TOB, IPM/CS }\end{array}$ & & 2.6 & Nonsurvival $^{\mathrm{a}}$ \\
\hline 8 & 81 & Male & $\begin{array}{l}\text { IPF, } \\
\text { aspergillosis }\end{array}$ & & & & CAM, VRCZ & LTOT & 1.3 & Nonsurvival $^{2}$ \\
\hline 9 & 74 & Male & MAC-PD & AS & & PPI & CAM & & 0.1 & Nonsurvivala \\
\hline 10 & 69 & Male & $\begin{array}{l}\text { COPD, MAC- } \\
\text { PD }\end{array}$ & & $\begin{array}{l}\text { Lobectomy for } \\
\text { pneumothorax }\end{array}$ & LAMA, LABA & & LTOT & 2.6 & Survival \\
\hline Median & 70.5 & & & & & & & & 1.8 & \\
\hline IQR & $\begin{array}{l}68.8 \\
\text { to } \\
75.8\end{array}$ & & & & & & & & $\begin{array}{l}0.9 \text { to } \\
2.8\end{array}$ & \\
\hline \multicolumn{11}{|c|}{${ }^{\mathrm{a}}$ Cause of death: Respiratory failure } \\
\hline \multicolumn{11}{|c|}{$\begin{array}{l}\text { MAC-PD: Mycobacterium avium complex pulmonary disease; LAMA: Long-acting muscarinic antagonists; LTOT: Long-term oxygen therapy; LAM: } \\
\text { Lymphangioleiomyomatosis, PH: Pulmonary hypertension; LABA: Long-acting } \beta 2 \text { agonists; NIV: Noninvasive intermittent ventilation; COPD: Chronic } \\
\text { obstructive pulmonary disease; ICS: Inhaled corticosteroids; COP: Cryptogenic organizing pneumonia; PPI: Proton pump inhibitors; BE: Bronchiectasis; } \\
\text { RFP: Rifampicin, STFX: Sitafloxacin; Transfer: Transfer to another clinic; IPF: Idiopathic pulmonary fibrosis; TOB: Tobramycin, CAM: Clarithromycin; HT: } \\
\text { Hypertension; EB: Ethambutol, LVFX: Levofloxacin; LC: Lung cancer; AZM: Azithromycin; RBT: Rifabutin; GC: Gastric cancer; IPM/CS: } \\
\text { Imipenem/cilastatin; GU: Gastric ulcer; VRCZ: Voriconazole; DM: Diabetes; AS: Ankylosing spondylitis }\end{array}$} \\
\hline
\end{tabular}


Table 2

Gastrointestinal symptoms, body weight status, breathlessness, and pulmonary function data in 10 inpatients with severe chronic respiratory illness

\begin{tabular}{|c|c|c|c|c|c|c|c|c|c|c|c|c|c|c|c|c|}
\hline \multirow[b]{2}{*}{$\begin{array}{l}\text { Case } \\
\text { No. }\end{array}$} & \multicolumn{4}{|c|}{$\begin{array}{l}\text { Gastrointestinal } \\
\text { symptoms }\end{array}$} & \multicolumn{5}{|l|}{ Appetite } & \multicolumn{7}{|c|}{ Breathlessness } \\
\hline & 1) & 2) & 3) & 4) & $\begin{array}{l}\text { VAS } \\
(\mathrm{mm})\end{array}$ & $\begin{array}{l}\text { BL } \\
\text { (cm) }\end{array}$ & $\begin{array}{l}\text { BW } \\
\text { (kg) }\end{array}$ & $\begin{array}{l}\text { Weight } \\
\text { loss } \\
(\%)\end{array}$ & BMI & mMRC & $\begin{array}{l}\text { During } \\
\text { eating }\end{array}$ & $\begin{array}{l}\text { Peripheral } \\
\text { edema }\end{array}$ & $\begin{array}{l}\text { FVC } \\
\text { (L) }\end{array}$ & $\begin{array}{l}\text { (\% } \\
\text { predicted) }\end{array}$ & $\begin{array}{l}\mathrm{FEV}_{1} \\
(\mathrm{~L})\end{array}$ & $\begin{array}{l}\text { (\% } \\
\text { predicted) }\end{array}$ \\
\hline 1 & & + & & & 72 & 31.6 & 3.7 & 13.5 & 3 & + & & 1.05 & 42.9 & 0.58 & 35.4 & \\
\hline 2 & + & + & & + & 81 & 158 & 48.3 & -1.3 & 18.6 & 4 & + & + & 1.14 & 41 & 0.32 & 15.8 \\
\hline 3 & + & + & + & & 43 & 164 & 36.2 & 0 & 13.5 & 4 & + & + & 1.89 & 54.3 & 0.58 & 19.2 \\
\hline 4 & & + & & + & 100 & 147 & 30.4 & 2.3 & 14 & 4 & + & + & 1.3 & 58.8 & 1.13 & 72.9 \\
\hline 5 & + & + & + & + & 48 & 167 & 41.7 & 9.6 & 15 & 4 & & + & 1.42 & 39.7 & 1.32 & 47.1 \\
\hline 6 & + & + & + & & 21 & 158 & 50.6 & 18.2 & 20.3 & 4 & + & + & 1.34 & 48 & 0.65 & 30.2 \\
\hline 7 & + & + & + & & 89 & 151 & 34.9 & 1.1 & 15.3 & 3 & + & & 1.06 & 46.4 & 0.64 & 37 \\
\hline 8 & + & + & + & & 39 & 169 & 53.6 & 7.1 & 19 & 4 & + & & 2.54 & 73.6 & 2.28 & 87.4 \\
\hline 9 & + & + & + & & 50 & 164 & 40.1 & 6.7 & 14.9 & 4 & + & & 1.22 & 35.3 & 1.16 & 42.8 \\
\hline 10 & & + & & & 43 & 169 & 47.5 & 23.4 & 16.6 & 3 & + & & 1.25 & 33.5 & 1.22 & 41.4 \\
\hline Median & & & & & 49 & 161 & 40.9 & 5.2 & 15.2 & 4 & & & 1.28 & 44.7 & 0.89 & 39.2 \\
\hline IQR & & & & & 42 to 83 & $\begin{array}{l}152 \\
\text { to } \\
167\end{array}$ & $\begin{array}{l}34.1 \\
\text { to } \\
49\end{array}$ & $\begin{array}{l}0.8 \text { to } \\
11.8\end{array}$ & $\begin{array}{l}13.9 \\
\text { to } \\
18.7\end{array}$ & 3 to 4 & & & $\begin{array}{l}1.12 \\
\text { to } \\
1.54\end{array}$ & $\begin{array}{l}38.6 \text { to } \\
55.4\end{array}$ & $\begin{array}{l}0.58 \\
\text { to } \\
1.25\end{array}$ & $\begin{array}{l}27.5 \text { to } \\
53.6\end{array}$ \\
\hline \multicolumn{17}{|c|}{$\begin{array}{l}\text { Gastrointestinal symptoms: 1) Early feeling of abdominal fullness, 2) Feeling of abdominal fullness and/or pain after meals, 3) Nausea, and 4) } \\
\text { Constipation }\end{array}$} \\
\hline
\end{tabular}

\section{Outcome}

Gastrointestinal symptoms, breathlessness during eating, and peripheral edema improved after acupuncture. Unresolved symptoms included the early feeling of abdominal fullness in one case, nausea in two cases, constipation in one case, and breathlessness during eating in two cases. Peripheral edema was not detectable in any case. Appetite loss significantly improved (VAS: 49 [IQR: 42 to 83] vs. 40 [IQR: 19.5 to 50.8 ] mm, p = 0.0039 ) and the amount of food intake significantly increased (1519 [IQR: 1204 to 1620] vs. 1653 [IQR: 1443 to 2047] Kcal/day, p = 0.0039) after nine acupuncture treatments over 3 weeks (Table 3). Dietary intake improved significantly (1523 [IQR: 1300 to 1626] vs. 1764 [IQR: 1483 to 2050] kcal/day, p = 0.0078), except for patient 6, whose daily dietary intake was limited to $1200 \mathrm{Kcal} /$ day due to diabetes. Body weight loss (0 [IQR: -1.4 to 1.75$] \mathrm{kg}, \mathrm{p}=0.0078,0$ [IQR: -3.25 to 4.7$] \%, p=$ 0.0078) 6 months after acupuncture was significantly improved compared to that documented 6 months before acupuncture (-3.8 [IQR: -6.4 to -0.3$] \mathrm{kg}$, -5.3 [IQR: -11.8 to -0.83 \%) (Fig. 1). The correlations among the differences in the VAS appetite scores, food intake (Kcal/day), body weight (kg), and BMI before and after acupuncture were as follows: food intake and appetite $(r=-0.6860, p=0.0285)$, body weight and appetite $(r=-0.6189, p=0.0564)$, and BMI and appetite $(r=-0.5714, p=0.0844)$, body weight and food intake $(r=0.6159, p=0.0580)$, and $B M I$ and food intake $(r=0.6322, p=0.0498)$. 
Table 3

Effects of 3-week acupuncture on appetite, food intake, and biomarkers.

\begin{tabular}{|c|c|c|c|c|}
\hline & $\begin{array}{l}\text { Pre-acupuncture } \\
\text { Median (IQR) }\end{array}$ & $\begin{array}{l}\text { Post-acupuncture } \\
\text { Median (IQR) }\end{array}$ & p-value* & $\begin{array}{l}\text { Normal reference } \\
\text { value }\end{array}$ \\
\hline Appetite (VAS: mm) & 49 (42 to 83 ) & 40 (19.5 to 50.8$)$ & 0.0039 & \\
\hline Food intake (Kcal/day) & 1519 (1204 to 1620$)$ & 1653 (1443 to 2047) & 0.0039 & \\
\hline Body weight (kg) & 40.9 (34.1 to 48.9$)$ & 41.1 (33.9 to 49$)$ & 0.6406 & \\
\hline BMI & 15.2 (13.9 to 18.7$)$ & 15.2 (14.5 to 18.7 ) & 0.6426 & \\
\hline \multicolumn{5}{|l|}{ Biomarkers } \\
\hline Albumin (g/dL) & 3.15 (3 to 3.8 ) & 3.4 (3.3 to 3.7 ) & 0.0293 & $4 \sim 5$ \\
\hline Prealbumin (mg/dL) & 11.35 (10 to 19.5$)$ & 15.2 (11.1 to 21.6$)$ & 0.0137 & $22 \sim 40$ \\
\hline $\mathrm{RBP}(\mathrm{mg} / \mathrm{dL})$ & 1.45 (1.2 to 2.2 ) & 1.9 (1.4 to 2.4$)$ & 0.0625 & $1.9 \sim 4.8$ \\
\hline WBC $(/ \mu \mathrm{L})$ & 5.57 (4.4 to 6.4$)$ & 4.99 (3.4 to 6.4$)$ & 0.418 & $3.5 \sim 8.5$ \\
\hline CRP (mg/dL) & $1.3(0.2$ to 3.7$)$ & 0.3 (0.1 to 2.3$)$ & 0.0977 & $0 \sim 0.3$ \\
\hline LDH (U/L) & 174 (159 to 210$)$ & 175 (149 to 205$)$ & 0.5156 & $119 \sim 229$ \\
\hline CPK (U/L) & 42 (20 to 66$)$ & 44 (28 to 55$)$ & 0.5078 & $45 \sim 163$ \\
\hline RDW & 13.9 (13 to 15$)$ & 14.0 (13.3 to 14.9 ) & 0.4141 & $11.8 \sim 15.5$ \\
\hline Epinephrine (pg/mL) & 41 (28 to 58$)$ & 40 (24 to 56$)$ & 0.6934 & $0 \sim 100$ \\
\hline Norepinephrine (pg/mL) & 728 (391 to 1286) & 591 (359 to 1015) & 0.375 & $100 \sim 450$ \\
\hline Dopamine $(\mathrm{pg} / \mathrm{mL})$ & 16 (7 to 36$)$ & 19 (11 to 25$)$ & 1 & $0 \sim 20$ \\
\hline Total ghrelin (fmol/mL) & 42.5 (26.9 to 88.5$)$ & 43.6 (18.6 to 65.2 ) & 0.0273 & \\
\hline Acyl ghrelin (fmol/mL) & 5.9 (3.7 to 12.6$)$ & 5.4 (3.2 to 12$)$ & 0.4961 & \\
\hline Des-acyl ghrelin (fmol/mL) & 35.7 (22.8 to 70.7 ) & 36.1 (14.7 to 15.6$)$ & 0.0293 & \\
\hline \multicolumn{5}{|l|}{ Urinary catecholamines } \\
\hline Epinephrine ( $\mu \mathrm{g} /$ day $)$ & 10 (9 to 17$)$ & 15 (8 to 20 ) & 0.5098 & $3.4 \sim 26.9$ \\
\hline Norepinephrine ( $\mu \mathrm{g} /$ day) & 226 (156 to 371$)$ & 182 (155 to306) & 1 & $48.6 \sim 168.4$ \\
\hline Dopamine ( $\mu \mathrm{g} /$ day) & 826 (631 to 1013$)$ & 1116 (611 to 1783$)$ & 0.2754 & $365 \sim 961.5$ \\
\hline
\end{tabular}

Serum levels of nutritional biomarkers (Alb, Pre-Alb) significantly increased after acupuncture (Table 3). A significant difference in the total ghrelin level was significantly correlated with a decrease in plasma norepinephrine level $(r=0.7455, p=0.0133)$. Although the levels of plasma norepinephrine and 24 -hour urinary norepinephrine were higher than the normal reference values before and after acupuncture, no significant decreases in both norepinephrine levels were observed post-treatment. The differences in 24-hour urinary norepinephrine levels before and after acupuncture were weakly correlated with the differences in daily dietary intake $(r=-0.6140, p=0.0590)$. Excluding Case 6 , whose daily dietary intake was restricted, they were significantly and inversely correlated with increased food intake $(r=-0.8954, p=0.0011)$. In addition, this difference was correlated with an increase in serum Alb levels $(r=-0.6544, p=$ 0.0401) (Fig. 2).

\section{Safety}

No treatment-related serious events were reported in the present study.

\section{Discussion}

All cachectic patients treated with acupuncture had advanced and severe chronic respiratory diseases with a poor prognosis. Six patients died within 3 years after 3 weeks acupuncture (survival time: 1.8 [IQR: 0.9 to 2.8] years). Five patients with refractory MAC-PD had undergone multi-drug combination chemotherapies several times; however, the results of mycobacterial smear tests from sputum specimens were always positive for clarithromycin-resistant acid-fast bacteria. Cases 2 and 6 with chylothorax had histologically diagnosed LAM on lung biopsy 18 and 21 years previously, respectively.

Acupuncture improved 1) breathlessness during eating, 2) gastrointestinal symptoms, and 3) peripheral edema. These results are likely to lead to significant improvements in appetite and dietary intake among patients with cachexia. The increase in food intake was significantly associated with improvements in 
appetite and BMI. Furthermore, nutritional biomarkers also improved significantly after acupuncture. Based on these results, short-term (nine times over 3 weeks) acupuncture safely improved the appetite and nutritional status of patients with advanced chronic respiratory illness and severe anorexia.

\section{Effects of acupuncture on breathlessness}

All participants felt short of breath during light exercises, such as eating, and had severely limited exercise capacity. These patients with advanced respiratory diseases display characteristics of exercise-induced hypoxemia and acidosis during daily activities [20-22]. This increase in exertional breathlessness indicates an elevation of plasma norepinephrine level during exercise [23]. In addition, participants had significantly higher levels of plasma norepinephrine and 24-hour urinary norepinephrine, as compared with normal reference values at rest (Table 3 ). These results indicated that the participants were always in a state of sympathetic nerve tone. Sympathetic hyperactivity weakens the peristaltic movements of the digestive tract and reduces the secretion of digestive enzymes and is considered to be one of the causes of gastrointestinal symptoms.

Acidosis and hypoxemia stimulate the solitary nucleus of the medulla oblongata (respiratory center), which sends ventilation-driving stimuli to the respiratory muscles, in order to maintain homeostasis. Patients with advanced respiratory illness require additional ventilation due to pulmonary gas exchange disorders, which are responsible for a further increase in dyspnea. The work of breathing is increased, resulting in excessive oxygen consumption [21]. Acupuncture results in reduced oxygen uptake and improved exertional dyspnea due to the improvement of oxygen utilization and reduced ventilation during exercise [11]. We considered that acupuncture reduced breathlessness by reducing exposure to hypoxemia, acidosis, and sympathetic hyperactivity in daily life, leading to an improvement in appetite.

\section{Effects of acupuncture on gastrointestinal symptoms}

The neural network that controls the balance between feeding and satiety mainly consists of the hypothalamus, including the paraventricular nucleus and the arcuate nucleus, and the solitary nucleus in the medulla oblongata. This network also affects feeding behavior. Acupuncture at ST36 afferently stimulates the solitary nucleus in the medulla oblongata and hypothalamus via the vagus nerve, and efferently improves peristalsis of the digestive tract [24, 25]. Further, this acupuncture point has been utilized for disorders of gastrointestinal function since ancient times. Acupuncture at ST36 affected the patient's feeding and satiety neural network and was thought to improve digestive peristalsis and enzyme secretion weakened by sympathetic hyperactivity. As a result, their gastrointestinal tract was emptied. Therefore, their food intake increased through appetite ameliorated by a natural process. Improvements in gastrointestinal function by acupuncture may have played a role in increasing nutritional biomarkers.

\section{Effects of acupuncture on appetite hormone (ghrelin) and catecholamine}

Acupuncture has been previously reported to provide benefits to patients with cancer or diabetic gastroparesis by reducing gastrointestinal symptoms, increasing gastric emptying, and altering physiological functions of anabolic/catabolic peptides (ghrelin and leptin) that play important roles in gastrointestinal functions [12-16]. Although circulating levels of ghrelin have been reported to be elevated in underweight patients with COPD [26, 27], levels of acyl ghrelin and des-acyl ghrelin in this study were not higher than those in elderly healthy volunteers in other studies [27]. Eight of 10 patients displayed sympathetic overactivity, as assessed by the levels of plasma norepinephrine and 24-hour urinary norepinephrine. The reduction in sympathetic overactivity correlated with the increase in food intake and serum Alb levels. However, improvements in food intake and nutritional biomarkers were observed among patients without sympathetic overactivity, whose des-acyl ghrelin levels decreased after acupuncture. This significant decrease in the des-acyl ghrelin level probably contributed to improvements in food intake and nutritional biomarkers [28]; however, it is possible that the cause and effect could be reversed. Alternatively, the change in plasma norepinephrine level was significantly correlated with the difference in plasma total ghrelin level before and after acupuncture. Ghrelin and catecholamines play an important role in the mechanism of improved dietary intake by acupuncture; however, it was difficult to clarify the mechanism in this preliminary study. Further studies with other biomarkers, such as appetite hormones (e.g., leptin and insulin) and inflammatory indicators (e.g., tumor necrosis factor) are warranted.

\section{Limitations}

First, the randomized trial is required to prove the potential effects of acupuncture, although the randomization of cachexia patients near death is ethically difficult. Second, due to the inclusion of patients with various types of respiratory disease, the heterogeneity of their medications, including chemotherapy, probably limits the interpretation of the results. Therefore, patient populations should be divided according to the presence or absence of chemotherapy intervention. Third, ghrelin and catecholamines may play important roles in the mechanism by which acupuncture improves dietary intake, but other biomarkers need to be added to further elucidate the mechanism.

\section{Conclusion}

Acupuncture could be a novel, safe, and beneficial treatment for anorexia and weight loss in cachexia patients with advanced respiratory diseases, although it is required to further validate the efficacy and investigate the mechanism.

\section{Abbreviations}

BMI : body mass index

COPD : chronic obstructive pulmonary disease

MAC-PD : Mycobacterium avium complex pulmonary disease 
IPF : idiopathic pulmonary fibrosis

LAM : Iymphangioleiomyomatosis

VAS : visual analog scale

CRP : C-reactive protein

Alb : albumin

Pre-Alb : prealbumin

IQR : interquartile range

\section{Declarations}

\section{Ethics approval and consent to participate}

This study was performed in accordance with the 1964 Declaration of Helsinki and its later amendments, the Guidelines for Good Clinical Practice for Epidemiological Studies, the Standards for Reporting Interventions in Clinical Trials of Acupuncture (STRICTA and STROBE), and the Guidelines for Clinical Research issued by the Japanese Ministry of Health. The institutional review board of the National Hospital Organization Osaka Toneyama Medical Center approved this study (number: 1580). Written informed consent was obtained from all individual participants in the study.

\section{Consent for publication}

Not applicable

\section{Availability of data and materials}

The datasets used and/or analyzed during the current study are available from the corresponding author on reasonable request.

\section{Competing interests}

The authors declare no potential conflicts of interest with respect to the research, authorship, and/or publication of this article.

\section{Authors' contributions}

TM, RM, and SK designed the study, collected the data, and wrote the paper. TM, RM, KM, MM, and SK recruited patients and collected the information.

TM and RM performed the statistical analysis; TM managed the data; TM and RM wrote, reviewed, and edited the manuscript. All authors read and approved the final manuscript.

\section{Acknowledgments}

The authors would like to express their deep appreciation to the patients who participated in the study, and the research staff (nutritionists, nurses, and laboratory technicians) who assisted during all stages of the study. We also thank Teppei Yamashita (Senior Lecturer, PhD) for the statistical analysis and Editage (www.editage.com) for the English language editing. This study received Grant-in-Aid for Clinical Research from the National Hospital Organization in Japan.

\section{Author information}

\section{Affiliations}

Department of Respiratory Medicine, National Hospital Organization Osaka Toneyama Medical Center, 5-1-1 Toneyama, Toyonaka, Osaka, 560-8552, Japan

Tomonori Maekura , Keisuke Miki , Mari Miki , Seigo Kitada , Ryoji Maekura

Graduate School of Medical Safety Management, Jikei University of Health Care Sciences, 1-2-8 Miyahara, Yodogawa-ku, Osaka 532-0003, Japan

Tomonori Maekura, Ryoji Maekura

\section{References}

1. von Haehling S, Anker MS, Anker SD. Prevalence and clinical impact of cachexia in chronic illness in Europe, USA, and Japan: facts and numbers update 2016. J Cachexia Sarcopenia Muscle. 2016;7:507-9.

2. Celli BR, Cote CG, Marin JM, Casanova C, Montes de Oca M, Mendez RA, et al. The body-mass index (BMI), airflow obstruction, dyspnea, and exercise capacity index in chronic obstructive pulmonary disease. N Engl J Med. 2004;350:1005-12. 
3. Hayashi M, Takayanagi N, Kanauchi T, Miyahara Y, Yanagisawa T, Sugita Y. Prognostic factors of 634 HIV-negative patients with Mycobacterium avium complex lung disease. Am J Respir Crit Care Med. 2012;185:575-83.

4. Kulkarni T, Yuan K, Tran-Nguyen TK, Young-II Kim, Joao A, de Andrade T, Luckhardt, et al. Decrements of body mass index are associated with poor outcomes of idiopathic pulmonary fibrosis patients. PLoS One. 2019;14:e0221905.

5. Nakatsuka Y, Handa T, Kokosi M, Tanizawa K, Puglisi S, Jacob J, et al. The clinical significance of body weight loss in idiopathic pulmonary fibrosis patients. Respiration. 2018;96:338-47.

6. Yoshimura K, Maekura R, Hiraga T, Miki K, Kitada S, Miki M, et al. Identification of three exercise-induced mortality risk factors in patients with COPD. COPD. 2014;11:615-26.

7. Wagner PD. Possible mechanisms underlying the development of cachexia in COPD. Eur Respir J. 2008;31:492-501.

8. Miki K, Maekura R, Nagaya N, Nakazato M, Kimura H, Murakami S, et al. Ghrelin treatment of cachectic patients with chronic obstructive pulmonary disease: a multicenter, randomized, double-blind, placebo-controlled trial. PLoS One. 2012;7:e35708.

9. Rabinovich RA, Ardite E, Mayer AM, Polo MF, Vilaro J, Argiles JM, et al. Training depletes muscle glutathione in patients with chronic obstructive pulmonary disease and low body mass index. Respiration. 2006;73:757-61.

10. Suzuki M, Muro S, Ando Y, Shiota T, Endo K, Sato S, et al. A randomized, placebo-controlled trial of acupuncture in patients with chronic obstructive pulmonary disease (COPD): the COPD-acupuncture trial (CAT). Arch Intern Med. 2012;172:878-86.

11. Maekura T, Miki K, Miki M, Kitada S, Maekura R. Clinical effects of acupuncture on the pathophysiological mechanism of chronic obstructive pulmonary disease during exercise. Int J COPD. 2019;14:2787-98.

12. Zhou J, Fang L, Wu WY, He F, Zhang XL, Zhou X, et al. The effect of acupuncture on chemotherapy-associated gastrointestinal symptoms in gastric cancer. Curr Oncol. 2017;24:e1-5.

13. Li G, Huang C, Zhang X, Xie H, Cheng H, Tang Y, et al. The short-term effects of acupuncture on patients with diabetic gastroparesis: a randomised crossover study. Acupunct Med. 2015;33:204-9.

14. Cabyoglu MT, Ergene N, Tan U. The mechanism of acupuncture and clinical applications. Int J Neurosci. 2006;116:115-25.

15. Gücel F, Bahar B, Demirtas C, Mit S, Cevik C. Influence of acupuncture on leptin, ghrelin, insulin and cholecystokinin in obese women: a randomised, sham-controlled preliminary trial. Acupunct Med. 2012;30:203-7.

16. Suzuki M, Muro S, Fukui M, Ishizaki N, Sato S, Shiota T, et al. Effects of acupuncture on nutritional state of patients with stable chronic obstructive pulmonary disease (COPD): re-analysis of COPD acupuncture trial, a randomized controlled trial. BMC Complement Altern Med. 2018;18:287.

17. Parker BA, Sturm K, MacIntosh CG, Feinle C, Horowitz M, Chapman IM. Relation between food intake and visual analogue scale ratings of appetite and other sensations in healthy older and young subjects. Eur J Clin Nutr. 2004;58:212-8.

18. Nagaya N, Itoh T, Murakami S, Oya H, Uematsu M, Miyatake K, et al. Treatment of cachexia with ghrelin in patients with COPD. Chest. 2005;128:118793.

19. Akamizu T, Murayama T, Teramukai S, Miura K, Bando I, Irako T, et al. Plasma ghrelin levels in healthy elderly volunteers: the levels of acylated ghrelin in elderly females correlate positively with serum IGF-I levels and bowel movement frequency and negatively with systolic blood pressure. J Endocrinol. 2006;188:333-44.

20. Kagawa H, Miki K, Kitada S, Miki M, Yoshimura K, Maekura R, et al. Dyspnea and the varying pathophysiologic manifestations of chronic obstructive pulmonary disease evaluated by cardiopulmonary exercise testing with arterial blood analysis. Front Physiol. 2018;9:1293.

21. Miki K, Maekura R, Hiraga T, Kitada S, Miki M, Yoshimura K, et al. Acidosis and raised norepinephrine levels are associated with exercise dyspnoea in idiopathic pulmonary fibrosis. Respirology. 2009;14:1020-6.

22. Miki K, Maekura R, Hiraga T, Kitada S, Miki M, Yoshimura K, et al. Exertional dyspnea-related acidotic and sympathetic responses in patients with sequelae of pulmonary tuberculosis. J Physiol Sci. 2010;60:187-93.

23. Maekura R, Hiraga T, Miki K, Kitada S, Yoshimura K, Miki M, et al. Differences in physiological response to exercise in patients with different COPD severity. Respir Care. 2014;59:252-62.

24. Takahashi T. Acupuncture for functional gastrointestinal disorders. J Gastroenterol. 2006;41:408-17.

25. Hui KK, Liu J, Marina O, Napadow V, Haselgrove C, Kwong KK, et al. The integrated response of the human cerebro-cerebellar and limbic systems to acupuncture stimulation at ST 36, as evidenced by fMRI. Neuroimage. 2005;27:479-96.

26. Itoh T, Nagaya N, Yoshikawa M, Fukuoka A, Takenaka H, Shimizu Y, et al. Elevated plasma ghrelin level in underweight patients with chronic obstructive pulmonary disease. Am J Respir Crit Care Med. 2004;170:879-82.

27. Zhang X, Yang T, Wang J, Feng M, Hou Y, Shen Y, et al. Elevated circulating ghrelin in patients with COPD: a meta-analysis. Chron Respir Dis. 2018;15:365-73.

28. Chen CY, Inui A, Asakawa A, Fujino K, Kato I, Chen CC, et al. Des-acyl ghrelin acts by CRF type 2 receptors to disrupt fasted stomach motility in conscious rats. Gastroenterology. 2005;129:8-25.

\section{Figures}




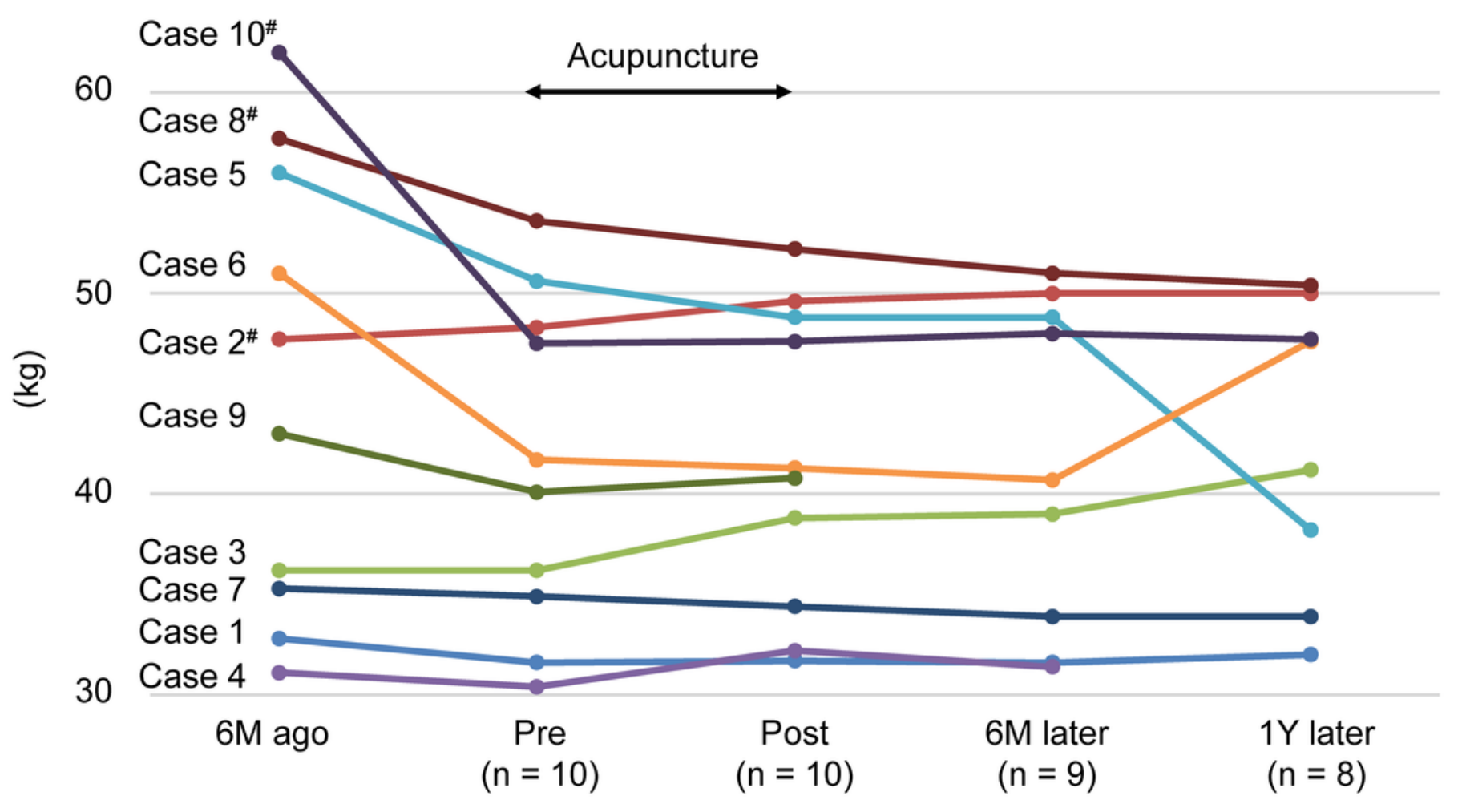

BW (kg): $45.3(31.1$ to 58.4$) \quad 41.5$ (34.1 to 48.9$) \quad 41.7$ (33.9 to 49.0$) \quad 41.7$ (33.1 to 49.4$) \quad 42.6$ (35.0 to 49.4 . $\begin{array}{lcl} & \\ -3.8[-6.4,-0.3] \mathrm{kg} / 6 \mathrm{M} & 0[-1.4,1.75] \mathrm{kg} / 6 \mathrm{M}^{*} & \text { Difference of BW }(\mathrm{kg}) / 6 \text { months } \\ -5.3[-11.8,-0.83] \% & 0[-3.25,4.7] \%^{*} & \text { BW loss }(\%) \text { of the last } 6 \text { months }\end{array}$ $*: p=0.0078$

Figure 1

Changes in body weight (BW) before and after acupuncture. Expressed in median (interquartile range), case \#: Acupuncture continued monthly.

a)

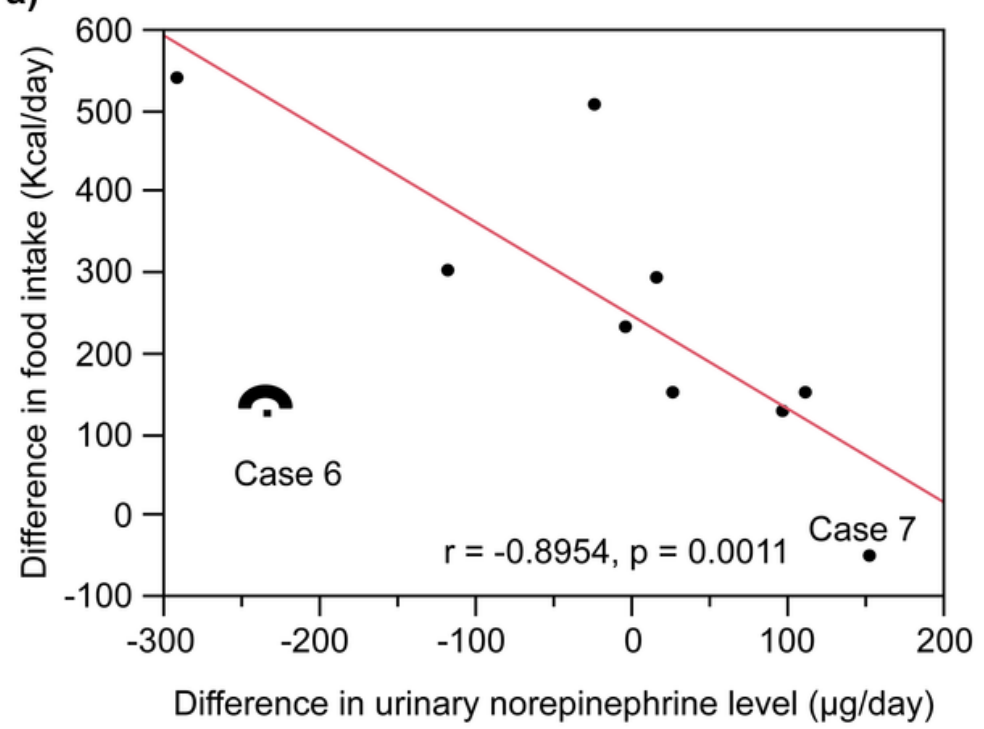

b)

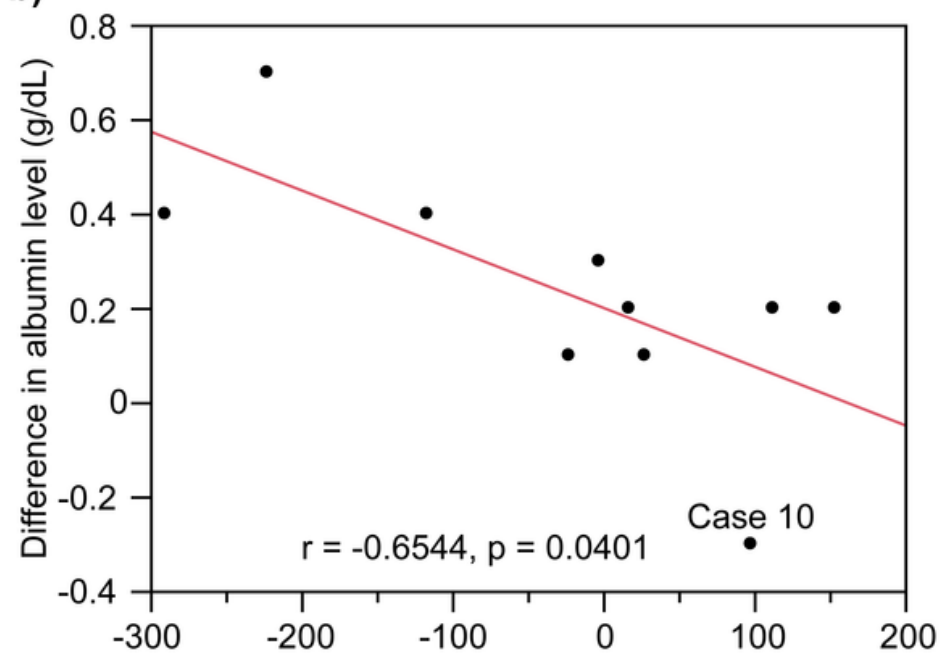

Difference in urinary norepinephrine level ( $\mu \mathrm{g} / \mathrm{day})$

Figure 2 
effects (gastrointestinal symptoms) were noted, and the patient complained of anxiety regarding her worsening condition. b) Case 10 continued monthly acupuncture treatment; the serum albumin level increased from $3.4 \mathrm{~g} / \mathrm{dL}$ to 3.9 and $4.2 \mathrm{~g} / \mathrm{dL}$ at 6 months and 1 year, respectively, after completion of the study. 